\title{
Spontaneous Disappearance of a Ruptured Intracranial Aneurysm
}

\author{
André Tokpa ${ }^{1}$, Louis Derouํ, Konan Serge Yaoㄹ, Dominique Oka N'dri² \\ ${ }^{1}$ Department of Neurosurgery, Teaching Hospital of Bouaké, Bouaké, Côte d'Ivoire \\ ${ }^{2}$ Department of Neurosurgery, Teaching Hospital of Yopougon, Abidjan, Côte d'Ivoire \\ Email:valentin_tokpa@yahoo.fr
}

How to cite this paper: Tokpa, A., Derou, L., Yao, K.S. and N'dri, D.O. (2019) Spontaneous Disappearance of a Ruptured Intracranial Aneurysm. Open Journal of Modern Neurosurgery, 9, 164-171. https://doi.org/10.4236/ojmn.2019.92016

Received: February 24, 2019

Accepted: April 15, 2019

Published: April 18, 2019

Copyright (c) 2019 by author(s) and Scientific Research Publishing Inc. This work is licensed under the Creative Commons Attribution International License (CC BY 4.0).

http://creativecommons.org/licenses/by/4.0/

(c) (i) Open Access

\begin{abstract}
The spontaneous disappearance of a ruptured intracranial aneurysm is unusual and its mechanism remains incompletely understood. However, several hypotheses are put forward and are mostly found in Virchow's triad. We report the case of a man who suffered subarachnoid hemorrhage by rupture of a blister aneurysm of the P1 segment of the left posterior cerebral artery. A control arteriography performed one week after the rupture showed a disappearance of the aneurysm and a significant vasospasm of the carrier artery. Angiograms performed at 3 weeks and 3 months confirmed this disappearance of the aneurysm and a return to normal artery size. Clinically the patient was doing well. Therefore his aneurysm was spontaneously declared cured. Several studies are needed to clarify the natural history of spontaneously thrombosed aneurysms and elucidate their occurrence mechanism in order to improve the management of intracranial aneurysmal pathology.
\end{abstract}

\section{Keywords}

Intracranial Aneurysm, Subarachnoid Hemorrhage, Cerebral Angiography, Spontaneous Thrombosis

\section{Introduction}

The spontaneous disappearance of a ruptured aneurysm is an unusual phenomenon even if it has been described several times [1] [2] [3] [4]. This disappearance may be temporary [5] or permanent. Thenceforth arises the problem of how to manage spontaneous thrombosis of a ruptured aneurysm. Moreover, even if several theories are put forward on the mechanism of this thrombosis, it remains unsolved. We report a case of spontaneous disappearance of a ruptured blister aneurysm from the posterior cerebral circulation and discuss its mechan- 
ism of occurrence as well as the action to be taken.

\section{Observation}

A 51-year-old patient complained of a sudden and bad headache. Secondarily, he has had a gradual deterioration of vigilance. He was hypertensive. He was admitted to the emergency room where the clinical examination revealed alertness disorder and meningeal stiffness. The Glasgow coma scale score was 13 . He had no sensory-motor deficit. The CT scan showed subarachnoid hemorrhage of grade 3 on the modified Fischer scale located predominantly in the prepontic cistern associated with tetraventricular hydrocephalus (Figure 1). The patient was transferred into the neurological intensive care unit. The cerebral angioscanner was normal. An external ventricular drainage was performed urgently followed by a cerebral arteriography. This last one reveals an aneurysm of the basilar tip, near the ostium of the left posterior cerebral artery, measuring 0.9 $\mathrm{mm}$ deep and $1 \mathrm{~mm}$ of cross-sectional diameter (Figure 2). A diagnosis of a ruptured blister aneurysm was made.

The procedure is stopped in the absence of immediate therapeutic possibility by simple coiling under satisfactory safety conditions (need for stenting \pm coiling involving antiplatelet preparation).

A multidisciplinary consultation meeting between neuroradiologist and vascular neurosurgeon is urgently held. They decided to carry out a cerebral angioscanner 3 days later and to schedule a new cerebral arteriography associated or not to endovascular treatment (stenting and coiling) in 1 week. A loading dose of antiplatelet will be necessary if stenting is indicated.

A cerebral arteriography of control is therefore performed one week later and made it possible to note the disappearance of the aneurysm initially identified. On the other hand, there is a diffuse spasm of the internal carotid arteries, the left anterior cerebral artery and the basilar trunk (which may mask aneurysm) (Figure 3).

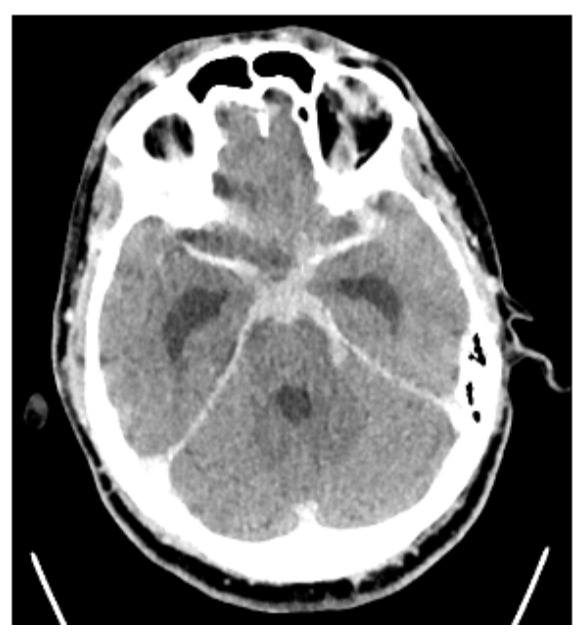

Figure 1. CT scan showing subarachnoid hemorrhage. 


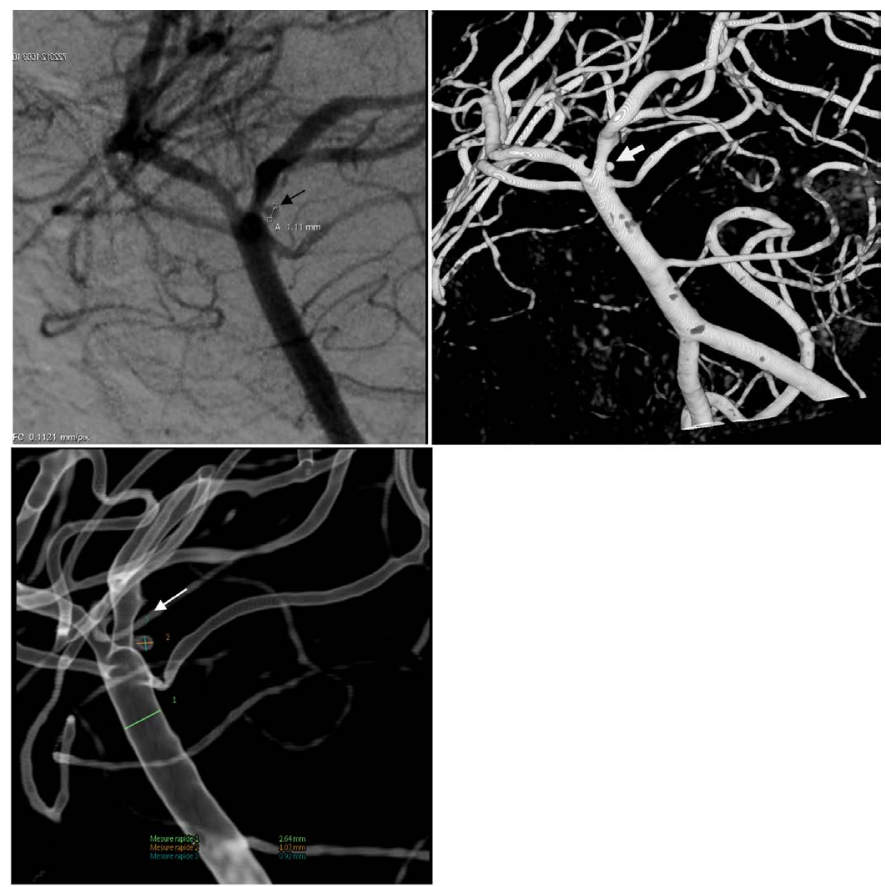

Figure 2. Arteriography showing a blister aneurysm (the arrows indicate the aneurysm).

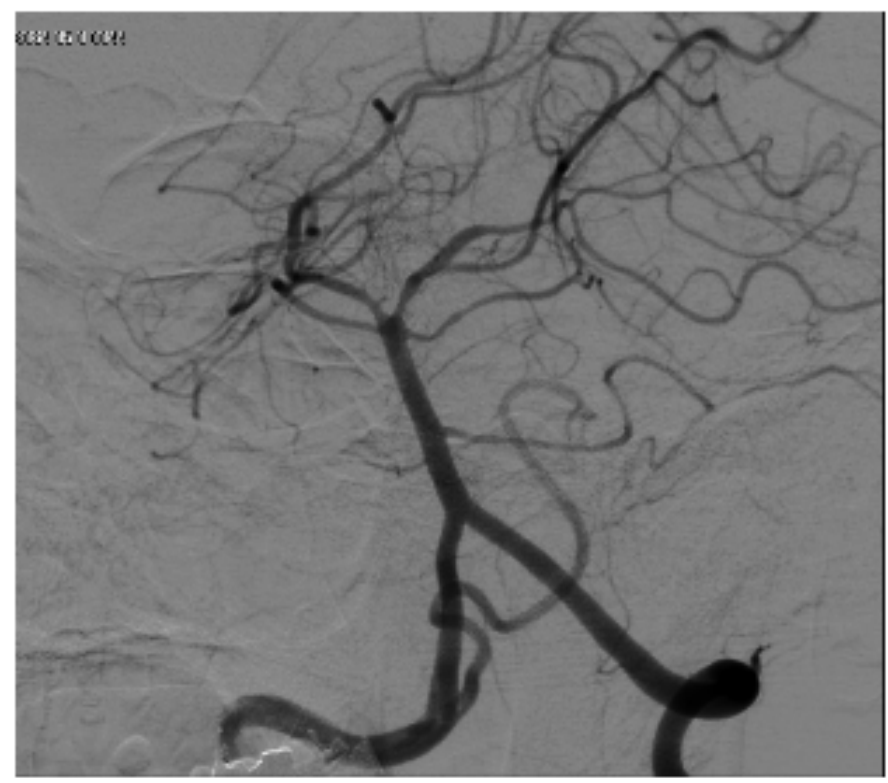

Figure 3. Arteriography showing vasospasm and disappearance of the aneurysm.

Given the asymptomatic nature of the spasm, no endovascular procedure is performed.

The patient's neurological condition gradually improves with decreased headache and confounding syndrome.

A brain MRI performed 2 weeks after the beginning of the symptomatology eliminated a parenchymal lesion; the spasm appears to be less important on this 
examination (subject to the technique's resolution limits). There is also no hydrocephalus.

The external ventricular drain is then clamped in the aftermath of the MRI, and removed 2 days later after a cerebral CT confirming the absence of hydrocephalus recurrence.

After validation of the absence of indication of ventriculoperitoneal shunting, the next step in the strategy will consist of stenting under dual antiplatelet therapy. The hypothesis of embolization by coiling-only of an aneurysm of this size is unlikely.

An attempt of treatment of the aneurysm was made. After administration of a loading dose of $20 \mathrm{mg}$ of prasugrel the day before, the procedure under general anaesthesia was performed and objectified a spontaneous thrombosis of the aneurysm and a good recovery of the size of the basilar artery and its branches, requiring no further treatment (Figure 4).

It was therefore decided to postpone endovascular treatment. The patient has been discharged. A control arteriography was performed 3 months later and confirms the spontaneous healing of the aneurysm (Figure 5).

\section{Discussion}

\subsection{Existence of the Phenomenon}

Thrombosis and spontaneous disappearance of giant aneurysms are well described [6], unlike small aneurysms for which the occurrence of spontaneous thrombosis is an unusual event [3]. However, this spontaneous thrombosis and disappearance of intracranial aneurysms does not seem to be uncommon in children [7] [8]. Spontaneous thrombosis of an intracranial aneurysm has been described in various autopsy series and occurred in $9 \%$ to $13 \%$ of cases [1]. The actual frequency of spontaneous thrombosis of intracranial aneurysms after

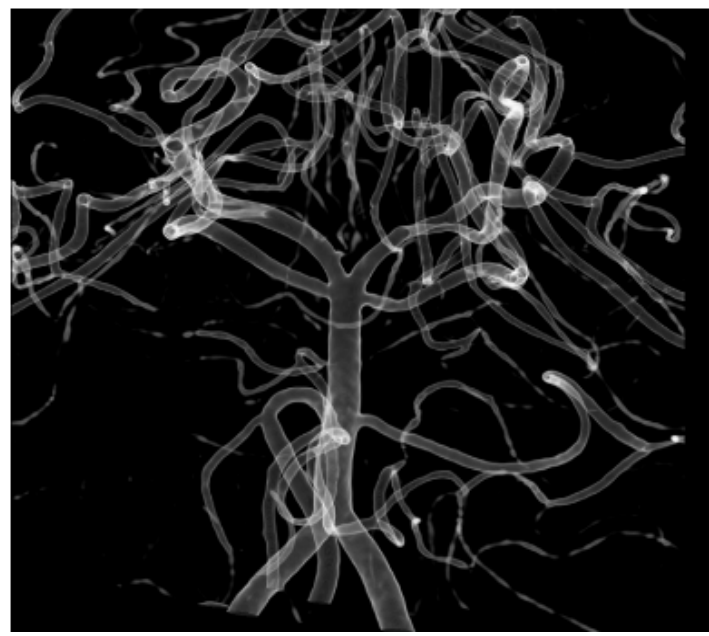

Figure 4. Arteriography showing the disappearance of the aneurysm and the normalization of the size of the carrier artery. 


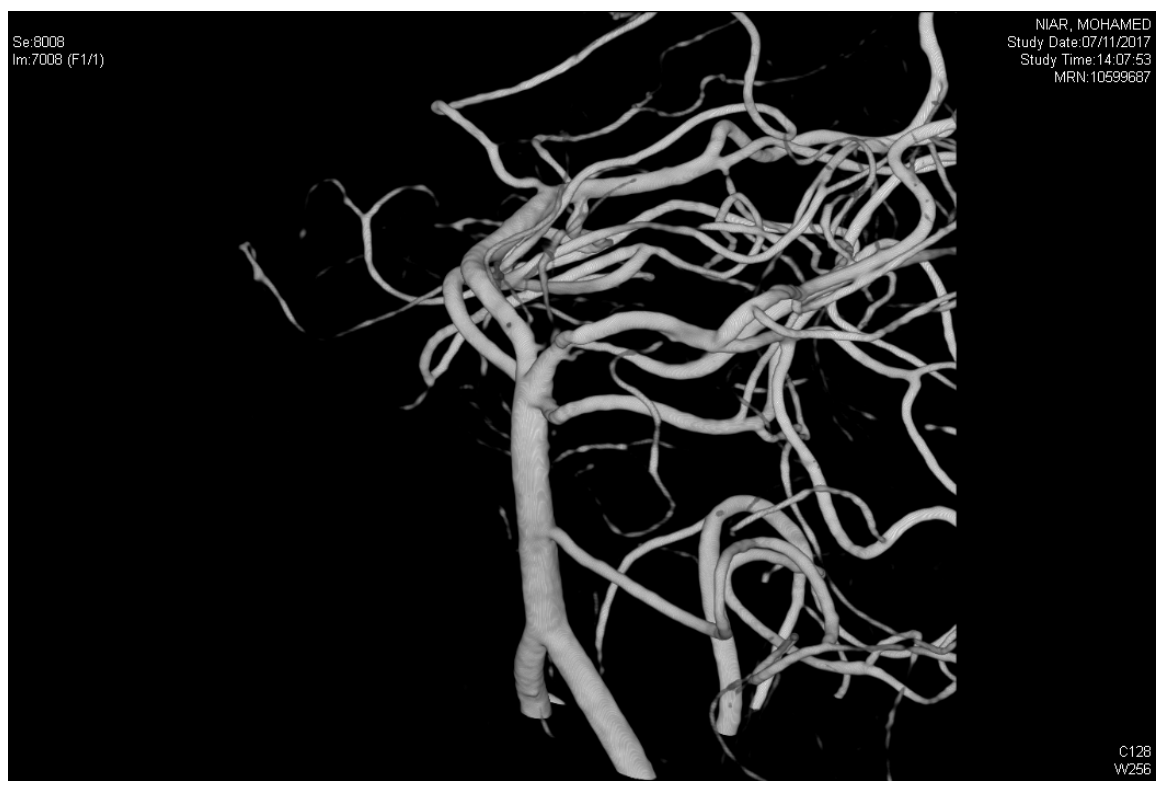

Figure 5. Arteriography showing the disappearance of the aneurysm 3 months after rupture.

subarachnoid hemorrhage is unknown. In 1945, Dandy suggested that perhaps $15 \%$ of patients with subarachnoid hemorrhage experienced spontaneous thrombosis and consequently healed [1]. Little and colleagues [9] observed in a series of 100 cases of subarachnoid hemorrhage with normal cerebral arteriography that in $5 \%$ of cases it was spontaneously thrombosed aneurysm.

\subsection{Mechanism of Spontaneous Thrombosis}

The exact mechanisms and factors responsible for this phenomenon have not yet been clarified. However, morphological, structural, hemodynamic and even drug-related factors would contribute to this phenomenon. Several hypotheses have been proposed to explain the mechanism underlying this phenomenon. Factors that promote spontaneous thrombosis include vasospasm [1], compressive effect of a surrounding hematoma [10], hypotension induced during anesthesia and cessation of blood flow during angiography [11]. It appears that most of these factors concern some aspects of Virchow's triad for vascular thrombosis: stasis, hypercoagulability and endothelial lesions [1]. Lasjaunias and colleagues believe that the arterial wall should be considered as a complex organ with a multitude of pathways leading to the formation and repair of aneurysms. In turn, these vascular wall destruction and repair mechanisms do not occur in isolation from the immediate perivascular environment. On the contrary, luminal and abluminal relationships should be considered as important biological and not only physical factors in intracranial aneurysmal vasculopathy [8].

Black and German [12] have shown that the volume/neck ratio of aneurysm is a major factor in the balance between thrombogenesis and thrombolysis. In case of aneurysm with a relatively narrow neck, intraluminal thrombosis may occur. In non-giant saccular aneurysms, a high volume to neck ratio and a low angle of 
inclination between the aneurysm and the parental artery has been recommended as factors promoting spontaneous thrombosis [13].

Schubiger and colleagues had suggested that a congenital defect in the tunica media of the vessel results in a change in hemodynamics and a turbulent flow causes clotting and deposition of onion-skin thrombotic layers. These intraluminal clots can reduce or, in extreme cases, completely obliterate the lumen of the aneurysm [14]. Pop and colleagues after having observed a spontaneous thrombosis of an aneurysm of the basilar artery tip following a ventriculoperitoneal shunting, estimated that 2 factors would have contributed to this thrombosis: the reduction of intracranial pressure and the modification (enlargement) of the angle formed by the aneurysm and the basilar artery [13].

Vasospasm can lead to low blood flow and temporary aneurysm thrombosis [15]. Hamilton and colleagues after observing a complete occlusion of a ruptured aneurysm, with permeability of all brain vessels confirmed by an angiogram performed twenty-four months after the rupture, considered that severe vasospasm, which complicated his subarachnoid hemorrhage, probably played a very important role in the thrombosis of this aneurysm [1].

Antifibrinolytic agents, particularly tranexamic acid, have been associated with the occurrence of spontaneous thrombosis of ruptured aneurysm [16]. Local inhibition of plasminogen activating factors in and around the aneurysm wall may cause spontaneous aneurysm thrombosis when treated with antifibrinolytic drugs.

In our patient, vasospasm seems to be the determining factor in the occurrence of thrombosis of his aneurysm. Incidentally, we could mention the involvement of blood factors promoting thrombogenesis and parietal factors. Moreover our patient having had a temporary external ventricular drainage, the decrease in intracranial pressure may have influenced the thrombosis of this aneurysm. It is quite clear that spontaneous thrombosis of a ruptured aneurysm is more than likely a multifactorial phenomenon [1].

\subsection{Management of Spontaneous Thrombosis of Ruptured Aneurysm}

Intra-aneurysmal thrombosis does not guarantee protection against further bleeding [6]. In addition, the thrombosed aneurysm can recanalize and break [4]. In 1974, Spetzler, and colleagues described a patient with severe hypertension and aneurysm of the left frontopolar artery initially present, who then disappeared and reappeared twenty-one days later [5]. For this reason, Davila and colleagues in 1984 suggested that spontaneous healing can only be described if three conditions exist: "a good neurological result; at least three months after the rupture and no cerebral infarction on CT" [17]. Therefore, a patient with a diagnosis of spontaneous thrombosis of an intracranial aneurysm should be followed by angiography at least 3 months after the onset of subarachnoid hemorrhage [1]. 


\section{Conclusion}

The occurrence of spontaneous thrombosis of a ruptured aneurysm may lead to its healing in some cases but sometimes secondary recanalization is possible; hence the interest of angiographic monitoring of these spontaneously thrombosed ruptured aneurysms. Precise knowledge of the mechanism of this spontaneous thrombosis could lead to new therapeutic approaches to aneurysmal disease. The evolution of the brain aneurysm should be considered as a dynamic process.

\section{Conflicts of Interest}

The authors declare no conflicts of interest regarding the publication of this paper.

\section{References}

[1] Hamilton, M.G. and Dold, O.N. (1992) Spontaneous Disappearance of an Intracranial Aneurysm after Subarachnoid Hemorrhage. Canadian Journal of Neurological Sciences, 19, 389-391.

[2] Jayakumar, P.N., Ravishankar, S., Balasubramaya, K.S., Chavan, R. and Goyal, G. (2007) Disappearing Saccular Intracranial Aneurysms: Do They Really Disappear? Interventional Neuroradiology, 13, 247-254. https://doi.org/10.1177/159101990701300304

[3] Nakau, H., Nagatani, H., Nakau, R. and Ametani, T. (2007) Acute Disappearance of Ruptured Aneurysm Located near the Origin of the Superior Cerebellar Artery-Case Report. Neurologia Medico-Chirurgica, 47, 468-470. https://doi.org/10.2176/nmc.47.468

[4] Su, T.M., Hsu, S.W., Chen, W.F., Lee, T.C. and Cheng, C.H. (2009) Acute Thrombosis and Recanalization of a Ruptured Anterior Communicating Artery Aneurysm. Journal of Clinical Neuroscience, 16, 1077-1079. https://doi.org/10.1016/j.jocn.2008.08.013

[5] Spetzler, R.F., Winestock, D., Newton, H.T. and Boldrey, E.B. (1974) Disappearance and Reappearance of Cerebral Aneurysm in Serial Arteriograms. Case Report. Journal of Neurosurgery, 41, 508-510. https://doi.org/10.3171/jns.1974.41.4.0508

[6] Whittle, I.R., Dorsch, N.W. and Besser, M. (1982) Spontaneous Thrombosis in Giant Intracranial Aneurysms. Journal of Neurology, Neurosurgery, and Psychiatry, 45, 1040-1047. https://doi.org/10.1136/jnnp.45.11.1040

[7] Buis, D.R., van Ouwerkerk, W.J., Takahata, H. and Vandertop, W.P. (2006) Intracranial Aneurysms in Children under 1 Year of Age: A Systematic Review of the Literature. Child s Nervous System, 22, 1395-1409. https://doi.org/10.1007/s00381-006-0142-3

[8] Lasjaunias, P., Wuppalapati, S., Alvarez, H., Rodesch, G. and Ozanne, A. (2005) Intracranial Aneurysms in Children Aged under 15 Years: Review of 59 Consecutive Children with 75 Aneurysms. Child s Nervous System, 21, 437-450. https://doi.org/10.1007/s00381-004-1125-X

[9] Little, A.S., Garrett, M., Germain, R., et al. (2007) Evaluation of Patients with Spontaneous Subarachnoid Hemorrhage and Negative Angiography. Neurosurgery, 61, 1139-1150. https://doi.org/10.1227/01.neu.0000306091.30517.e7 
[10] Cohen, J.E., Itshayek, E., Gomori, J.M., Grigoriadis, S., Raphaeli, G., Spektor, S. and Rajz, G. (2007) Spontaneous Thrombosis of Cerebral Aneurysms Presenting with Ischemic Stroke. Journal of the Neurological Sciences, 254, 95-98. https://doi.org/10.1016/j.jns.2006.12.008

[11] Kim, H.J., Kim, J.H., Kim, D.R., et al. (2014) Thrombosis and Recanalization of Small Saccular Cerebral Aneurysm: Two Case Reports and a Suggestion for Possible Mechanism. Journal of Korean Neurosurgical Society, 55, 280-283. https://doi.org/10.3340/jkns.2014.55.5.280

[12] Black, S.P.W. and German, W.J. (1960) Observations on the Relationship between the Volume and the Size of the Orifice of Experimental Aneurysms. Journal of Neurosurgery, 17, 984-990. https://doi.org/10.3171/jns.1960.17.6.0984

[13] Pop, R., Chibarro, S., Manisor, M., Proust, F. and Beaujeux, R. (2016) Spontaneous Thrombosis of a Basilar Tip Aneurysm after Ventriculoperitoneal Shunting. Journal of NeuroInterventional Surgery, 8, e34. https://doi.org/10.1136/neurintsurg-2015-011841.rep

[14] Schubiger, O., Valavanis, A. and Wichmann, W. (1987) Growth-Mechanism of Giant Intracranial Aneurysms; Demonstration by CT and MR Imaging. Neuroradiology, 29, 266-271. https://doi.org/10.1007/BF00451765

[15] Bohmfalk, G.L. and Story, J.L. (1980) Intermittent Aneurysm on Appearance Sequential of a Ruptured Angiograms. Journal of Neurosurgery, 52, 263-265. https://doi.org/10.3171/jns.1980.52.2.0263

[16] Fodstad, H. and Liliequist, B. (1979) Spontaneous Thrombosis of Ruptured Intracranial Aneurysms during Treatment with Tranexamic Acid (AMCA). Report of Three Cases. Acta Neurochirurgica, 49, 129-144. https://doi.org/10.1007/BF01808955

[17] Davila, S., Oliver, B., Molet, J. and Bartumeus, F. (1984) Spontaneous Thrombosis of an Intracranial Aneurysm. Surgical Neurology, 22, 29-32. https://doi.org/10.1016/0090-3019(84)90223-4 